\title{
Comment on "Shear modulus of two-dimensional Yukawa or dusty-plasma solids obtained from the viscoelasticity in the liquid state"
}

\author{
Sergey A. Khrapak $\oplus^{1,2}$ and Boris A. Klumov ${ }^{2}$ \\ ${ }^{1}$ Institut für Materialphysik im Weltraum, Deutsches Zentrum für Luft- und Raumfahrt, 82234 Weßling, Germany \\ ${ }^{2}$ Joint Institute for High Temperatures, Russian Academy of Sciences, 125412 Moscow, Russia
}

(Received 31 October 2019; accepted 1 May 2020; published 20 May 2020)

\begin{abstract}
In a recent paper [Wang, Huang, and Feng, Phys. Rev. E 99, 063206 (2019)], Langevin dynamical simulation results related to the shear modulus $G$ of a two-dimensional liquid dusty plasma were reported. The purpose of this Comment is to provide a comparison with available theoretical models to calculate $G$ and to indicate the correct way of interpreting the numerical results.
\end{abstract}

DOI: 10.1103/PhysRevE.101.057201

In a recent paper Wang et al. reported Langevin dynamical simulations of two-dimensional (2D) liquid dusty plasmas performed to investigate their shear modulus $G$ in the liquid and solid states [1]. Dusty plasma has been approximated by 1024 particles contained in a rectangular box and interacting via the pairwise Yukawa (screened Coulomb) potential of the form $\phi(r)=\left(Q^{2} / r\right) \exp (-r / \lambda)$, where $Q$ is the particle charge and $\lambda$ is the plasma screening length. Such a system is fully characterized by two dimensionless parameters: the coupling parameter $\Gamma=Q^{2} / a T$ and the screening parameter $\kappa=a / \lambda$, where $a=(\pi \rho)^{-1 / 2}, \rho$ is the particle density, and $T$ is the temperature in energy units. One of the conclusions they made is that the potential (excess) component of the infinite-frequency shear modulus of the Yukawa liquid is very close to the shear modulus of the 2D Yukawa solid at the same screening parameter. For Yukawa solids they used a phenomenological fit

$$
G(\kappa)=\left(Q^{2} / a^{3}\right)\left(0.211-0.0389 \kappa^{1.11}\right)^{2},
$$

based on the previous results from Refs. [2,3]. Unfortunately, no comparison with the available theoretical results on the high-frequency elastic moduli of two-dimensional Yukawa fluids was provided. The purpose of this Comment is to report such a comparison.

The high-frequency (instantaneous) elastic moduli of simple monatomic fluids can be related to the pairwise interaction potential $\phi(r)$ and radial distribution function (RDF) $g(r)$. A thorough analysis of the three-dimensional case was performed by Zwanzig and Mountain [4]. The 2D shear modulus can be expressed as $[5,6]$

$$
G_{\infty}=\rho T+\frac{\pi \rho^{2}}{8} \int_{0}^{\infty} d r r^{2} g(r)\left[3 \phi^{\prime}(r)+r \phi^{\prime \prime}(r)\right] .
$$

The first term on the right-hand side corresponds to the kinetic contribution and the second term is the potential (excess) contribution. This excess contribution dominates at strong coupling.

Generally, the RDF $g(r)$ is required as an input for the calculation of elastic moduli. For Yukawa systems the situation can be somewhat simplified. In the weak and moderately screened regime $\kappa \lesssim O(1)$, the $\operatorname{RDF} g(x ; \kappa, \Gamma)$ is not very sensitive to $\kappa[5,7]$ (here $x$ is the reduced distance $x=r / a$ ). This allows the shear modulus to be expressed in terms of the excess energy and its first and second derivatives with respect to $\kappa$ [5]. In addition, in the considered regime of $\kappa$, the excess energy is dominated by the static temperature-independent contribution, which can be adequately approximated by the corresponding lattice sum. This is a general property of soft repulsive potentials, which has been extremely well elaborated in the context of Yukawa fluids [8-10]. In this way the Madelung coefficient $M$ of triangular lattice becomes a quantity which determines the shear modulus. If the reduced lattice energy $U_{\mathrm{L}}$ per particle is expressed as a product of $M$ and $\Gamma, U_{\mathrm{L}} / N T=M \Gamma$, the instantaneous shear modulus reads $[5,11]$

$$
G_{\infty}=\rho T\left[1+\frac{\Gamma}{8}\left(\kappa^{2} \frac{\partial^{2} M}{\partial \kappa^{2}}+\kappa \frac{\partial M}{\partial \kappa}-M\right)\right]
$$

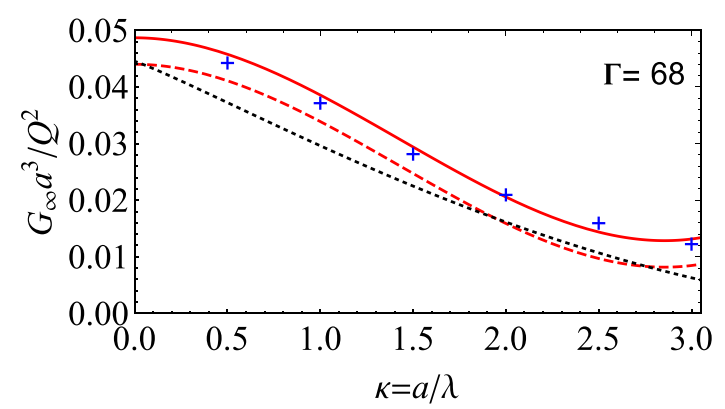

FIG. 1. Reduced instantaneous shear modulus $G_{\infty} a^{3} / Q^{2}$ of a 2D Yukawa fluid versus the screening parameter $\kappa$ at $\Gamma=68$. Symbols correspond to the results from numerical simulations [1], the red solid curve is the theoretical prediction of Eq. (3), and the red dashed curve corresponds to the excess contribution, i.e., Eq. (3) without the kinetic term. The black dashed curve is the fit of Eq. (1). 


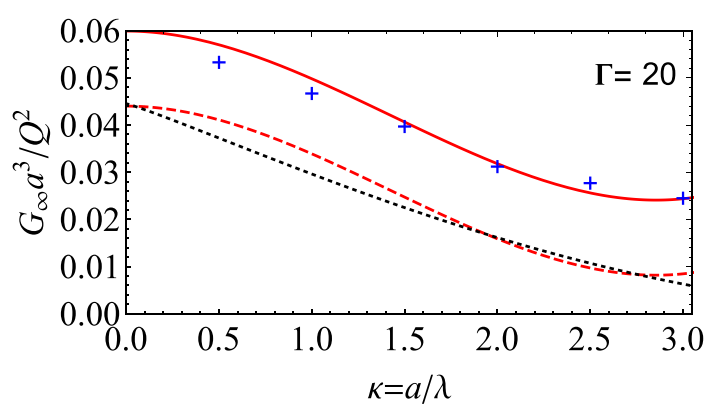

FIG. 2. Same as in Fig. 1 but for $\Gamma=20$.

The last step is to specify the dependence $M(\kappa)$. In Ref. [9] the expression (fit)

$$
M=-1.1061+0.5038 \kappa-0.11053 \kappa^{2}+0.00968 \kappa^{3}+\frac{1}{\kappa}
$$

was suggested, which was later used in Refs. [5,10]. This allows one to evaluate $G_{\infty}$ in a very extended range of $\kappa$ and $\Gamma$. Note that the approach just outlined is a good approximation for the strongly coupled fluid state and becomes exact in the limit of an ideal crystalline lattice. In the latter case the RDF (representing a series of $\delta$ peaks) is fixed and hence does not depend on $\kappa$ and the excess energy is exactly the lattice sum. A related observation is that the instantaneous shear moduli of a strongly coupled fluid and ideal lattice (at the same values of $\Gamma, \rho$, and $T$ ) are different only by the kinetic contribution, which is relatively small at strong coupling.

A comparison between the theoretical calculation and the numerical results from Ref. [1] for the instantaneous shear modulus of 2D Yukawa fluids is shown in Figs. 1-3 for three values of $\Gamma(\Gamma=68,20$, and 8$)$ and a weak to moderate screening regime $0 \leqslant \kappa \leqslant 3.0$. The instantaneous shear modulus is normalized by $Q^{2} / a^{3}$ following Ref. [1] (note that $\rho T a^{3} / Q^{2}=1 / \pi \Gamma$ ). The agreement between theory and experiment is rather good. The red dashed curve corresponds to the potential (excess) contribution in Eq. (3), which is a

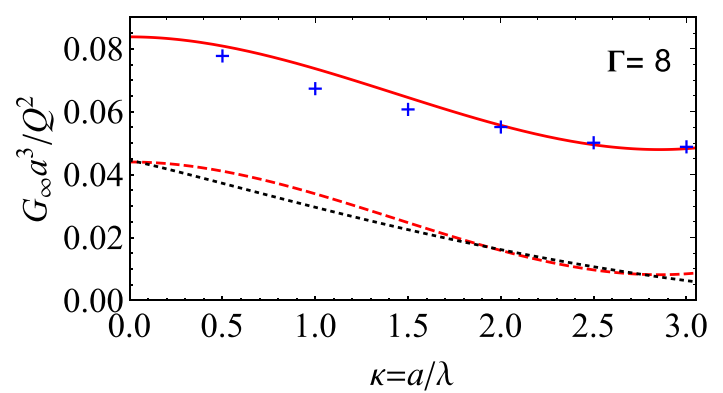

FIG. 3. Same as in Fig. 1 but for $\Gamma=8$.

proxy for the shear modulus of the 2D hexagonal Yukawa lattice. The fit of Eq. (1) is relatively close, but clear deviations are observable.

The following remarks can be added. The kinetic contribution to the instantaneous shear modulus is $\rho T$ and is numerically equal to $1 / \pi \Gamma$ when the chosen normalization is used. We get $G_{\infty}^{\text {kin }} \simeq 0.0047,0.0159$, and 0.0398 for $\Gamma=68,20$, and 8 , respectively. This is in very good agreement with the results presented in Figs. 4 and 5 of Ref. [1]. The role of the kinetic term decreases as coupling increases, as expected. The instantaneous shear modulus of the one-component plasma (OCP) limit $(\kappa=0)$ is well known. The quasilocalizedcharge approximation $[12,13]$ yields, for a strongly coupled 2D OCP, $G_{\infty} \simeq-\frac{1}{8} \rho T M \Gamma$, in full agreement with Eq. (3). Numerically, this results in $G_{\infty} a^{3} / Q^{2} \simeq 0.0440$ in the OCP limit (the neutralizing background has to be added, which cancels out the term $1 / \kappa$ in the Madelung coefficient and results in $\left.M_{\mathrm{OCP}} \simeq-1.1061\right)$. This coincides exactly with the Bonsall-Maradudin result for the hexagonal OCP lattice $[14,15]$. Equation (1) produces a close value of $G_{\infty} a^{3} / Q^{2} \simeq$ 0.0445 at $\kappa=0$. Finally, the fit of Eq. (4) is applicable for $\kappa \lesssim 3.0$ and should not be employed for stronger screening regimes.

The work leading to this publication was partly supported by the German Academic Exchange Service with funds from the German Aerospace Center. We would like to thank Volodimir Nosenko for a critical reading of the manuscript.
[1] K. Wang, D. Huang, and Y. Feng, Phys. Rev. E 99, 063206 (2019).

[2] F. M. Peeters and X. Wu, Phys. Rev. A 35, 3109 (1987).

[3] S. Nunomura, J. Goree, S. Hu, X. Wang, and A. Bhattacharjee, Phys. Rev. E 65, 066402 (2002).

[4] R. Zwanzig and R. D. Mountain, J. Chem. Phys. 43, 4464 (1965).

[5] S. Khrapak and B. Klumov, Phys. Plasmas 25, 033706 (2018).

[6] S. A. Khrapak, N. P. Kryuchkov, and S. O. Yurchenko, Phys. Rev. E 97, 022616 (2018).

[7] R. T. Farouki and S. Hamaguchi, J. Chem. Phys. 101, 9885 (1994).

[8] S. A. Khrapak, I. L. Semenov, L. Couëdel, and H. M. Thomas, Phys. Plasmas 22, 083706 (2015).
[9] I. L. Semenov, S. A. Khrapak, and H. M. Thomas, Phys. Plasmas 22, 114504 (2015).

[10] N. P. Kryuchkov, S. A. Khrapak, and S. O. Yurchenko, J. Chem. Phys. 146, 134702 (2017).

[11] S. Khrapak, J. Chem. Phys. 148, 146101 (2018).

[12] K. I. Golden and G. J. Kalman, Phys. Plasmas 7, 14 (2000).

[13] S. A. Khrapak, B. Klumov, L. Couëdel, and H. M. Thomas, Phys. Plasmas 23, 023702 (2016).

[14] L. Bonsall and A. A. Maradudin, Phys. Rev. B 15, 1959 (1977).

[15] S. A. Khrapak, N. P. Kryuchkov, L. A. Mistryukova, A. G. Khrapak, and S. O. Yurchenko, J. Chem. Phys. 149, 134114 (2018). 